A Dynamic, Multidimensional Approach to Knowledge Production

\author{
Ryan Light \\ University of Oregon \\ $\&$ \\ jimi adams \\ University of Colorado Denver
}

[Preprint Manuscript. For final version, please refer to Chapter 6 in Investigating Interdisciplinary Collaboration: Theory and Practice across Disciplines, Rutgers University Press 2016. https://www.rutgersuniversitypress.org/investigating-interdisciplinarycollaboration/9780813585888 ]

Please direct all correspondence to Ryan Light, Department of Sociology, University of Oregon, 1291 University of Oregon, Eugene, OR 97403-1291, light@uoregon.edu. 
Interdisciplinarity implies a resistance to the traditional boundaries structuring academic research. This unboundedness is inherently temporal: After all, if an interdisciplinary project sticks around long enough, it runs the risk of evolving into what it seeks to resist (i.e., a traditional discipline). Contemporary debates on the best social organization for addressing pressing scientific and/or social problems provide a valuable step away from the nearly universal push towards interdisciplinarity (Jacobs and Frickel 2009, Jacobs 2013). While funding continues to pour towards interdisciplinary organizations and strategies within universities, critical approaches to interdisciplinarity may help clarify exactly when a problem demands solutions generated outside of disciplinary boundaries. To proponents the promise of interdisciplinarity rests on efforts to transcend disciplinary boundaries to address complicated problems (Campbell 1969, Klein 1990): Disciplines are overly specialized and cannot respond to the multidimensional problems of our day. To opponents the danger of interdisciplinarity consists of a massive reshuffling of resources with limited benefit: Disciplines provide an effective social organization for the production of knowledge, especially given the specialization required for addressing difficult contemporary problems. Navigating these opposing positions requires a critical lens capable of tracing the production of knowledge through its various forms.

Recent research places greater emphasis on the temporal dimension of interdisciplinarity by recognizing that the organization of knowledge is a dynamic process (Jacobs 2013, Leydesdorff and Schank 2008). Moving beyond static discussion of whether interdisciplinarity or disciplinarity is the best organization for a particular problem, critical approaches to interdisciplinarity have argued that the worst case scenario described above is likely: Interdisciplinary projects turn into disciplinary projects as power brokers construct and manage boundaries to their benefit. This process mirrors the social closure common in status hierarchies 
as groups battle over limited resources. As seemingly benign interdisciplinary projects gain greater attention, they seek greater rewards, such as financial compensation for faculty and more graduate student labor. Interdisciplinary projects build an organization - often resembling a discipline - to garner and protect these rewards. While this tendency towards organization and resource hoarding may be true, the dynamic process is hidden by an over-determined trajectory. We propose softening this determinism to consider more seriously the boundary work that takes place during knowledge production.

In this chapter, we build a dynamic model of academic research that focuses on how research changes based on the characteristics of the boundaries that separate academic fields. Interdisciplinarity, disciplinarity, multidisciplinarity, and transdisciplinarity become particular potential states in the dynamic life course of a research field.[1] These different states hold implications for practitioners whose work is bounded by the characteristics of a particular state, at a particular time. In addition we emphasize that boundary work is a dynamic process: An analysis of the boundaries of knowledge production requires a focus on how boundaries are challenged and how they change. For example, a research field may shift, like American Studies or Biochemistry, from an interdisciplinary project to a more disciplinary one and interdisciplinary research may grow less visible as a result (Jacobs 2013; Kohler 1982). The shape of the trajectory that a knowledge project assumes at a given moment in time is important for evaluating its status as a many-disciplined project. Considering the life course of a knowledge project is key to the development of a critical lens for analyzing knowledge production.

Knowledge production takes place multidimensionally. Individuals compete for resources within organizations or groups; organizations or groups are embedded in fields. While these 
dimensions overlap and reinforce one another, they are analytically separable. In sum, we offer a multidimensional life course model of knowledge production. We begin by developing a theoretical model that hinges on the role of boundary work in structuring knowledge production. We specify boundary work as a dynamic process that shapes the life course of a knowledge project. Next, we describe how this life course perspective operates across the multiple levels of analysis from the individual project to the field. To illustrate this theoretical model, we tease apart several empirical trajectories spanning both projects and fields, including research on religion, demography, and HIV/AIDS. Conclusions illustrate the importance of developing robust critical approaches to interdisciplinarity grounded in comprehensive theoretical and methodological tools.

\section{Developing the Model}

\section{Many Disciplined Boundary Work}

Prior work advances a typology of knowledge production that consists of four categories. These categories - disciplinarity, multidisciplinarity, interdisciplinarity, and transdisciplinarity are often presented as static characteristics of knowledge projects. A static view likely overemphasizes the stability of many-disciplined approaches to knowledge production. The danger of this overly static approach is that highly organized projects may be able to cash in on the language of many disciplined projects without actually engaging in any cross-pollination between disciplines. To reorient this static perspective, research has turned to the boundary metaphor (Jacobs 2013). Boundaries within knowledge production exist along multiple dimensions. The first wave of research on boundaries identifies the lines drawn between science and the public (Gieryn 1999). The second wave of research on boundaries observes the boundaries drawn between groups within science itself (Jacobs 2013, Lamont and Molnar 2002). One of the key characteristics of the boundary framework is the acknowledgment that the lines 
drawn between groups - either within science or between science and the public - are produced and maintained with a good deal effort, or boundary work.

While boundary work takes multiple forms, its primary dimensions involve expulsion and expansion (Gieryn 1999). Expulsion occurs within knowledge production as actors, such as scientists, defend their turf against rivals and, at times, concede questions that are beyond reach or seem less worthy of defense. Expansion, on the other hand, consists of efforts to colonize a new research area - either one that is already the focus of a rival group or an otherwise unexplored territory. For example, physicists engage in expansion when turning their attention to macro social scientific problems, such as the study of large-scale social networks (see Newman 2008), while social scientists engage in expulsion when they try to resist this potentially cooptive move by physicists by pointing out redundancies or reinventions by the new network specialists (See Freeman 2004, 168).[2] Beyond offering a compelling narrative structure for internecine academic wars, boundary work provides a key mechanism for evaluating many disciplined projects as new problems are subject to strategic engagement by interested actors. This final logic motivates proponents of interdisciplinarity who see contemporary social and scientific problems as - at their core - requiring the attention of multiple approaches spanning several disciplines (Taylor 2010). Rewards are extended to projects that meet these criteria. Yet, as Jacobs (2013) points out, boundary work does not stop here, but continues past the distribution of initial rewards as expulsion and expansion efforts continue.

Towards a Dynamic Multidimensional Model of Knowledge Production

Our theoretical model takes this dynamic boundary work as its starting point and assumes that knowledge production consists of multiple strategies for engaging boundary-crossing. We focus on two particular dimensions: group- and individual-level motivations for crossing boundaries. Group motivations recognize that formally organized groups, such as pre-existing 
disciplines, will develop strategies focused on expansion and expulsion within fields. These motivations might be resource-based (i.e. struggling for financial rewards, lab space, graduate students) or idea-based (i.e. struggling to be a leader addressing a particular issue). Alternatively, individual motivations recognize that boundary work occurs at a more micro-scale as individual people and projects perform boundary tasks. Within an individual project, such as a research paper, scientists may engage multiple disciplines or may bound their arguments within a single discipline.

<Figure 6.1 about here>

Figure 6.1 summarizes our theoretical approach. The model distinguishes the project or individual dimension on the $\mathrm{y}$-axis and the group dimension on the $\mathrm{x}$-axis. Both of these dimensions vary by level of boundary crossing indicating whether these types of boundary work are priorities or not. These dimensions provide a comprehensive framework for considering the aforementioned types of organization - disciplinarity, multidisciplinarity, interdisciplinarity, and transdisciplinarity. Disciplines, for example, focus on boundary maintenance and, therefore, are generally less directed towards boundary crossing at the group or individual level. Consistent with Jacobs and Frickel's (2009) definition, however, multidisciplinary projects organize around group boundaries as multiple disciplines contribute to a research problem. Group boundary crossing is likely to be a priority, but is unlikely to result in integrated outcomes.

Interdisciplinarity, on the other hand, prioritizes these integrated efforts at the individuallevel as knowledge from two or more disciplines is intertwined to generate a new outcome, such as a patent or research paper. This integration can take place by a single author building expertise in multiple disciplines or from a group of disciplined scientists working together. Note that interdisciplinary projects may have some interest in group boundary crossing as well, especially 
as they become more organized; however, our model focuses on primary motivations or priorities. Last, transdisciplinarity projects represent a fundamental challenge to existing structures of knowledge as they call for a more radical reorganization with high boundary crossing at both the group and the individual level. Transdisciplinarity may serve to dramatically reduce the boundaries between the public and science (Frodeman 2014, Hadorn, Pohl and Bammer 2010) or effectively eliminate the boundaries between groups previously organized by disciplines.

Importantly, these states of organization are not permanent, but can be said to represent stages in the life course of a knowledge project. These stages indicate an empirically identifiable moment in time, but are characteristic of temporally embedded strategies by actors working on research problems. Again, these strategies of boundary work can lead to changes in organization. A life course perspective helps account for this temporal dimension by emphasizing the dynamics of knowledge production. Life course perspectives, borrowed from interdisciplinary work on health and aging, are resolutely longitudinal. In his overview of criteria for establishing a life course perspective, Mayer (2009) states that a key characteristic of life course research is a commitment to understanding change in human lives "over a long stretch of the lifetime" and not narrow windows of time. In the life course of knowledge projects, it is problematic to speak solely about brief - and often arbitrary - moments in time. While it may be empirically valuable to observe the consequences of the establishment of an interdisciplinary research center in the near term, the story of a knowledge project does not end here, but continues beyond these key moments. For example, as resources accrue around this hypothetical center it may assume the appearance locally of a discipline-based department (e.g. awarding degrees and hiring faculty). Not a discipline in a clear sense, the center may also grow to discourage interdisciplinary 
interaction within the university as an attempt to preserve boundaries and scarce resources. A dynamic life course model acknowledges these shifting strategies and fates.

Studying the life course of humans presents, on its face, a well-bounded span: Human lives begin and end at specific moments in time. The boundedness of knowledge projects is less specific. For example, whether emerging Frankenstein-like from the combination of previous knowledge projects or more subtly through fractalization (Abbott 2001), the starting-point of a knowledge project is often more difficult to determine. This ambiguity presents a challenge to anyone studying the dynamics of knowledge projects. While our model does not resolve this issue, a life course approach to knowledge production requires some attention to start- and endpoints. These points may be determined in numerous ways, but deserve careful consideration and clear articulation. In this way, the life course perspective that we propose may be more like how humans often describe their cultural versus their biological lives as a series of rebirths, new core turning points and so forth. In a similar vein, those using our perspective should look for these key moments of transition as identifiable temporal boundaries.

Life course perspectives also are resolutely multidimensional: As Mayer (2009) writes, change is "studied across life domains." When studying human lives, crossing domains means the consideration of multiple aspects of the lived experience from family to work to health. Within the life course of a knowledge project, crossing domains requires a theoretical position that allows for the consideration of multiple aspects of knowledge production from field-level efforts at boundary maintenance to resource-based struggles at the organizational-level, to problem-based contestation over ideas. This multidimensionality contrasts with prior work on interdisciplinarity which tends to define knowledge production along one dimension, while subordinating others. As both Jacobs (2013) and Frodeman (2014) identify, for example, most 
prior definitions focus on epistemological - or problems-based - dimensions of interdisciplinarity at the expense of resources or politics. In other words, work that romanticizes the combination of core disciplinary perspectives often misses the material struggle taking place between actors and organizations.

Our theoretical approach embraces the multidimensionality of the life course perspective by accounting for knowledge production at the field, organization, and problem-level. By requiring a multilevel consideration, our model suggests that prior perspectives based largely on either resources or idea-based considerations require integration for establishing a complete theoretical story about interdisciplinarity. While data limitations may focus attention on one dimension at the expense of others, critical approaches to interdisciplinarity should attempt to construct a multidimensional and dynamic lens for engaging knowledge production. In fact, the life course of some knowledge projects likely includes an uneasy relationship between stage alignment across those levels. For example, the tension that occurs when a rival interdisciplinary approach turns away from a problem-based challenge to an established discipline to struggle over resources (e.g. neuroscientists demanding more lab space from their psychology colleagues or gender studies scholars arguing for departmental resources).

To highlight the dynamics of knowledge production, we focus our attention on trajectories of knowledge projects. Trajectories are paths across the stages identified in Figure 1. These trajectories can follow many paths. For example, we can observe how a knowledge project moves from interdisciplinary research towards a disciplinary structure. Alternatively, we can observe projects that were solved and/or abandoned prior to the establishment of an organizational structure. 
Consider the case of American Studies as described by Jacobs (2013) (see also Dubrow 2011). The trajectory of American Studies begins with an epistemological complaint: The humanities in the United States were too wedded to European thought and offered little space to domestic cultural products, literary and otherwise. Initial boundary work focuses on expansion into the under-explored waters of American culture and an attempt to re-historicize literary studies given the mid-Twentieth Century dominance of ahistorical New Criticism. Mid-century historians also had their complaints about the rigidity of the dominant approach to historical study that focused on key figures. Early work in American Studies, thus, begins in a multidisciplinary vein. In ensuing decades, American Studies expands to include some of the resources characteristic of disciplines - including approximately 150 undergraduate degree programs by 1968 - yet it remained largely multidisciplinary (i.e. the degree programs drew faculty from the English and History departments).

By the 1990s, even despite sluggish undergraduate enrollment, 40 universities offered advanced degrees in American Studies and 6 universities had stand-alone PhD-granting departments. The ideas-based expansion resolved to organizational struggles for recognition within universities. Obviously this turn from epistemological to organizational concerns was only very modestly successful. At the field-level, American Studies has experienced more success with thriving journals and well-attended annual meetings preserving an interdisciplinary spirit. As Jacobs $(2013,186)$ writes, "In its efforts to transcend disciplinary boundaries, a fully interdisciplinary approach to academia would create a dizzying array of alternative maps of the intellectual terrain.” American Studies obviously didn't accomplish this type of radical restructuring; however, its organizational failures may have helped preserve its interdisciplinarity. 
The life course of American Studies to this point includes a trajectory from a multidisciplinary approach to boundary expansion through organizational struggles that mirror disciplinarity to interdisciplinary field-level structures. From an ideas-based perspective the history of American Studies may seem resolutely successful, while from a resources-based perspective its failure to grow its campus presence may be deemed a failure. By encouraging multidimensionality, a life course lens allows for a more mixed story by accommodating both ideas- and resource-based explanations. As such, the case of American Studies highlights the need for a dynamic, multidimensional approach to knowledge production. In the following section, we illustrate the multidimensional life course approach by focusing on the shifting boundaries of knowledge production for three cases: social sciences of religion, demography, and several specific problems within HIV/AIDS research.

\section{Exemplar Trajectories}

To illustrate the efficacy of the model developed above, in the section that follows, we provide a number of empirical examples that demonstrate how particular projects have evolved through the various stages of boundedness that correspond to forms of (many) disciplinarities. While many trajectories are possible, we focus on several that we have identified as common in empirical work that has attempted to account for field and/or problem level dynamics. In the description above, we note how disciplinary projects can define and/or cross boundaries variously at the levels of fields, organizations and problems. The examples below focus particularly on: (1) field-level trajectories that either (a) begin from or (b) end in disciplinary forms, and (2) problem-level trajectories for questions that are (a) resolved or (b) remain open. This leaves open the possibility for empirical investigations into how organizational structures evolve across the states identified by our model. While the parameters of the model would 
sufficiently allow for examining such questions, we simply don't currently have data to investigate that level within the projects we draw from here. As such, we invite future researchers to investigate how readily the forms described in the model, and the trajectories demonstrated in the examples below are found in organizational settings.

A Field that Began as a Discipline - Social Sciences of Religion

The Society for the Scientific Study of Religion was founded in 1949 with its primary contributors coming from theology backgrounds (Newman 1974). While these founders were motivated by questions pertinent to theology and religious practice, they identified a need for perspectives from across the (social) sciences to best examine those questions (Newman 1974). To examine how readily these fundamentally interdisciplinary aims translated into practice, and how the structure of the field has evolved in the six decades since its formation, we draw on a corpus of 41,186 articles.[3] With these articles, we construct a series of coauthorship networks, which draw ties between individuals based on their having written one (or more) papers together (Yan and Ding 2012). These coauthorship networks can then be analyzed to identify network communities that reveal groups of researchers who comprise the boundaries between "invisible colleges" (or thought collectives) within research fields (Gmur 2003).

The evolution of these clusters is estimated over the last six decades, starting just after the formation of the field as described above. These clustering patterns evolve across the period revealing four distinct configurations; the shift between these different organizational patterns of the field happen to each roughly correspond to a decade. The earliest period was one of relatively little systematic organization, with mostly scattershot, disconnected research projects that had limited communication-directly, who collaborates with whom, or indirectly, through sharing similar intellectual forebears. As the field grew, it rapidly evolved into a small but highly consolidated research field by the end of the 1970s. This signature is consistent with the 
ideological origins of the organization - an interdisciplinary (social) scientific study of religion. This changed relatively rapidly however - through most of the 1980s, this initial enthusiasm appears to have waned somewhat, as the growth in the number of publications plateaued. In addition, the early consolidation was superseded by an increasing bifurcation within the literature. This separation into distinct communities was further entrenched into the 1990s, and even expanded to move from two into multiple communities. By the first decade of the 2000s, this segmentation became even greater, with those identifiable communities exhibiting even stronger boundaries between them.

A comparison of this last period - one of relatively entrenched boundaries - to a similar analysis of the whole of social sciences conducted by Moody and Light (2006) helps to make sense of the source of this evolution. The left panel of Figure 6.2 presents the bibliographic coupling network among papers published from 2000-2009 in the social sciences of religion corpus used here. The labels overlaid come from our examination of the authors on a sample of the most central papers within each cluster. What is revealed in comparing these clusters identified in our analysis (left panel of Figure 6.2) to those from Moody and Light's (2006, Figure 1 p. 71) analysis (right panel) is that the resulting clusters are remarkably similar in content and arrangement to those exhibited across all social science publications. That is, the field appears to have evolved to currently mimic the general structure exhibited within the social sciences.

$<$ Figure 6.2 about here>

The trajectory of the social sciences of religion is therefore one that progressed from initial disciplinary motivations, through relatively strong interdisciplinary collaboration, into a 
strongly multidisciplinary state where researchers from across the social sciences appear to be contributing to the content of the field, but doing so mostly within the confines of their own disciplinary homes. We don't want to overstate the strength of these boundaries - there is some interaction across these boundaries, but the prevalent pattern here is one that is much more segmented than some of the other patterns that will be described below. One potential explanation for this emergent structure is the perspective offered by Smilde and May (2010) that our understanding of religion has matured into a "strong program" with distinctly measurable characteristics of religiosity that are increasingly understood to have identifiable causes and effects of importance to a range of social scientific interests.

Fields that Evolved into a Discipline - The Demography Example

Demography's origins lie primarily in a number of population concerns - whether aims to control population growth globally or for particular populations, or to promote fertility or migration patterns to enhance goals of particular societies (Hodgson 1991). From the outset, these concerns drew interest from a wide range of social sciences (Liu and Wang 2005) and were represented by core philosophical, mathematical and statistical contributions (Kreager 1993). Given this orientation, one would expect clustering patterns that align with those key research questions - and that is what is observed in the examples below - clusters that primarily oriented, separately, around fertility, mortality and migration research topics. This early problem orientation crossed disciplinary boundaries, and provided meaningful organization as participants in the field sought to address related sets of concerns. Of particular interest for our purposes here is how that organization subsequently changed as demographic questions increasingly coalesced into entrenched scientific questions, methods and findings. Did it retain the early interdisciplinary organization, or evolve into some alternative pattern? As time passed, did the relationship(s) between each of the three key processes in demographic research change? 
To assess this, rather than the coauthorship networks used above, we combine two approaches using five decades of publications from five leading demography journals.[4] First, we construct a series of bibliographic coupling networks, which draw ties between papers based on the degree of overlap between the references they cite (Yan and Ding 2012). These bibliographic coupling networks can then be analyzed in the same manner as above to identify network communities. These communities reveal groups of research papers that rely on highly similar bodies of literature to construct their arguments, an alternative strategy for discovering "invisible colleges" within research fields (Gmur 2003). We then combine this network clustering with an analysis of the content of these research clusters. We do this by examining the frequency of the articles' identified keywords within each cluster. For most of the observed period (1956-2012), the field is structured primarily around three dominant clusters, which remain relatively closely aligned separately with the three focal demographic processes (i.e, fertility, mortality and migration). Other topics periodically emerge (and wane); leading to a question of how the arrangement changes through time - both among the three core topics and those other more short-lived themes.

The early period of these journals is marked by research on the "demographic transition," or the effects of industrialization on demographic outcomes which is marked by increasing life expectancy, decreasing infant mortality and later declining fertility (Hodgson 1991) with a corresponding close overlap between research simultaneously engaging mortality and fertility. Over time, the strength of the boundaries between each of these three topics increases, but the boundaries never fully separate these literatures from one another: The central position in the literature moves from being relatively equally occupied by fertility and mortality topics to being occupied solely by fertility research. Additionally, migration literature starts out as and remains 
more peripheral than either of the others throughout the period. Most of the secondary topics observed in the period (e.g., family planning, environmental consequences of population growth, and HIV/AIDs - each prominently featuring within this sample of the demographic literature for slightly more than a decade) arise as topics that primarily bridge between the fertility and mortality clusters and have little connection to the migration topic.

This combination of patterns is consistent with an orientation that began largely around a single question (population growth) with a consolidated transdisciplinary arrangement. Over time, three primary research topics developed and retained distinct community boundaries. While occasional crossing occurred between them, bridging most likely took place via unaligned or secondary research topics. As a result, the field remains structured by primary communities: fertility, mortality, and migration. Along with this trajectory, there has been the founding of a professional association, journals, and even degree programs, leading some to identify demography as a discipline unto itself demarcated by the conceptual and methodological tools available for addressing these three topics (Morgan and Lynch 2001). The disciplinary boundaries around demographic research however, remain relatively permeable with frequent contributions within those topically oriented clusters from scholars from a number of disciplines, including sociology, economics, public health, and others. This trajectory therefore raises the question of what would allow us to identify when transdisciplinary research fields (those with high individual and project level boundary crossing) coalesce strongly enough to qualify as a new discipline. This is a topic of much previous research (Bettencourt et al. 2008, Rojas 2010), and something we think would be an important integration for future applications of the model we develop here.

Resolved Problem-Oriented Questions in HIV/AIDS Research 
In much of the literature extolling the potential benefits of interdisciplinary work, a key assumption is that approaches drawing from ideas spanning disciplinary boundaries will be more likely to generate solutions (Boyack, Klavans and Börner 2005, NAS et al. 2005). In some cases, this has proven to be correct. Drawing from our work on HIV/AIDS research (adams and Light 2014, Light and adams 2010), we identify a few topics that incorporate key resolved questions, and describe the trajectories of those cases across the model described above. Our contention is that if we map out several of these trajectories, it can allow us to shift the conversation from an oversimplified question of "Does interdisciplinary research benefit solving problems?" to a more theoretically useful question of "What patterns of disciplinary boundary crossing allow for more efficient problem solving?" In other words, these help us focus on the process(es) that underpin successes than on the successes themselves. This is helpful not only for making sense of the focal questions themselves, but also for thinking about how these models can potentially be of use to other cases.

Few recent research fields have witnessed a more public and urgent call to interdisciplinarity than HIV/AIDS research. HIV/AIDS was initially conceived as a problem with important contributions to be made by medical practitioners, epidemiologists, policy makers, and activists, among other constituencies. In the case of HIV/AIDS research, two key empirical developments with wide-ranging implications have been the development of nevirapine - a single dose treatment used to prevent mother-to-child transmission (PMTCT) of HIV at birth, and the development of strategies for testing for HIV antibodies.

In this case, like the demography example above, we align bibliographic network clusters with topics. Instead of using keywords for identifying the content, we use examples from our work elsewhere that use Topic Models (adams and Light 2014, Light and adams 2010). This 
approach consists of a class of techniques that locate structure in unstructured text corpora (Blei Griffiths and Steyvers 2004, Steyvers and Griffiths 2007) by "reverse engineering" the writing process to uncover latent themes within the corpus that underlie their generative processes.

Topics models locate patterns within text data based on how words overlap in texts, in this case scientific abstracts. While the math operating behind topic models is quite complicated, the logic is simple: Some words appear together with greater likelihood than others. By identifying these distributions of words - or topics - we can develop an overview of what scientists have written about in our collection of abstracts. By using unstructured data, topic models include more finegrained information than using other bibliometric data, such as keywords. While many methods are needed to successfully explore knowledge projects, topic models may be particularly important for locating emergent themes as constructed by scientists themselves without relying on cumbersome predefined or user-generated coding schemes.[5] Within the topic models of HIV/AIDS abstracts, PMTCT and the development of testing assays were each identified as among 30 primary topics present in the first 20 years of published work in AIDS and JAIDS, two many-disciplined journals on HIV/AIDS.

The PMTCT of HIV became a focus in the field at a relatively early period in the field of HIV/AIDS research, especially as work increasingly focused on Africa (which became an overrepresented topic in the field beginning in the late 1990s). Single dose nevirapine became available in the early 2000s, which reduces the likelihood of mother-to-child transmission at birth by over $40 \%$ (Guay et al. 1999). As such, describing how the prevention of mother-to-child (or PMTCT) literature progressed within the HIV/AIDS field can provide an informative case. By extracting only those papers that engaged the PMTCT topic out of the bibliographic coupling networks described above, we examined the community patterns of this literature through time, 
and illustrate its evolution in Figure 6.3 (color represents section labels from the journal blue=basic science, red=clinical, yellow $=$ social/epidemiological sciences $)$.

$<$ Figure 6.3 about here>

What this topic demonstrates is an early period in which there was a single consolidated research community, but within that community, researchers were represented from a wide range of disciplinary backgrounds. This is consistent with the pattern of transdisciplinarity as described above - virtually boundaryless work attempting to collectively solve a problem of practical importance. Over time, this pattern changed - most drastically, in the periods after the availability of nevirapine (2001 and following). Namely, while this topic remains relatively well consolidated in a single cluster, some minor segmentation arises (see split between the north and western portions in the 2008 slice), and this consolidated cluster became increasingly dominated only by researchers from the social science.[6] This is a move that is increasingly consistent with a more disciplinary organization - perhaps consistent with the practical concern of moving from attempting to identify how to prevent MTCT to a focus on removing barriers to implementing that identified solution. In sum, the trajectory of PMTCT is one of high transdisciplinary integration that led to the discovery of nevirapine which resolved a particular aspect of the problem, and has lead subsequently to a topic largely addressed within disciplinary confines.

Research on the development of reliable testing assays for identifying when someone has HIV was a central question in the field that arose almost immediately after the identification of HIV as the putative causal agent of AIDS. The literature on this topic within the journals AIDS and JAIDS followed a similar structural pattern to that of PMTCT described above in that it arose and remained consolidated in a single research community, until reliable methods were available for wide use (Delaney et al. 2006). However, this literature differed from the PMTCT trajectory 
in two important ways. First, the consolidated cluster associated with addressing this question had a remarkably variable composition, represented by bench scientists attempting to develop the actual assays, and social scientists more concerned with the potential usability of the developed solutions: This case was marked more by interdisciplinary organization early in the observed period. Once a solution was identified, the contribution of this research topic to the corpus became substantially diminished and almost eliminated completely by the late 2000 s. Open Problem-Oriented Questions in HIV/AIDS Research Not all forms of many disciplined science are created equally. While the differences between the various forms described above have been well documented, it's also plausible to identify problems that exist in similar states identified by the model that exhibit differing levels of success in addressing the questions they raise. As with above, our question at this stage is not necessarily to equate particular forms and/or trajectories as being more/less likely to generate successes or failures for problem oriented science. We aren't to that stage yet. Instead we are simply describing problems that exhibit particular trajectories and--for now--seeking to identify commonalities and/or differences between those. In this section, we continue to focus on examples from our work on HIV/AIDS aligning bibliographic clustering networks and topic models, but instead turn to trajectories that are observed for questions that remain, as yet, unresolved. In particular, we examine the trajectories of topics pertaining to three research areas: drug failures especially those that arise from the development of drug resistance, the pursuit of an HIV vaccine, and efficacy of antiretroviral treatment. These three examples exhibit substantially different trajectories.

$<$ Figure 6.4 about here> 
The drug resistance topic we identified from the AIDS/JAIDS corpus is a topic that does not have any substantial contribution in the literature until the early 2000s. Once it arises, it does so with representation in two separate network communities. Those two communities remain prominent and separated from one another throughout the 2000s. Moreover, the split between these two communities appears to be more aligned with disciplinary divisions than with problem-oriented differentiation. As in the example presented in Figure 6.4, one of the communities addressing this topic is more closely aligned with the bench/basic sciences, while the other is more closely aligned with clinical-based research. This alignment fluctuates moderately across the observed period, but the general pattern is one of dominance within those segmented clusters that differentiate more along paradigmatic (disciplinary) orientations throughout. Drug resistance research has been resolutely multidisciplinary within AIDS and JAIDS.

Research on vaccines is marked by a substantially different structural signature from the drug resistance literature, but exhibits a similarly remarkable stability in that structure over time. Vaccine development is a research topic that features relatively prominently throughout the history of the journals $A I D S$ and JAIDS. Moreover, it is a literature that appears relatively consolidated into a single community across that entire period. Where it differs is in the composition of that community - being dominated almost exclusively by basic science research-predominantly virologists who specialize in vaccine research. HIV vaccine development appears to have remained a disciplinary pursuit for three decades.

Research on the efficacy of antiretroviral treatments exhibits substantially different trajectories than either of the other "open" questions described above. First, like drug resistance research, it is a relatively late-breaking research topic (showing little presence in the early-mid 
1990s). Once it arises, however it does so within a relatively consolidated single community-mostly associated with bench sciences (likely the same researchers who were concerned with developing the treatments in the first place). Over time, this research topic has increasingly segmented into coverage by two separate research communities. Those separate communities are increasingly (in the mid-late 2000s) represented by clinical research concerned with evaluating the effectiveness of treatment regimens, which is separate from more epidemiological research that is concerned with identifying differential predictors (particularly the social determinants) of the success/failure of those treatment regimens. In sum, over time this research topic has increasingly been further segmented into differential primary research questions, which are increasingly independently addressed by researchers from different disciplinary perspectives; i.e., has evolved from a mostly disciplinary approach to a more multidisciplinary orientation. Conclusions and Future Directions

Knowledge production does not follow a predetermined path. Prior theoretical attention to interdisciplinarity assumes many disciplined projects follow one of two trajectories. Interdisciplinary utopians see interdisciplinarity as the skeleton key to solving complex, pressing problems, while skeptics worry that interdisciplinarity obscures resource-based contests. Our approach attempts to avoid these overly determined trajectories by building dynamics into the core of knowledge production. When viewed from a life course perspective, stages of research such as disciplinarity and interdisciplinarity, are not static, but are subject to change.

As our examples illustrate, knowledge projects may occur along different trajectories with shifting boundaries as the projects mature and as problems resolve or remain open. From a field-based perspective, the social scientific study of religion grew out of disciplines - religious studies and theology - through a process of expansion as the social sciences grew in prominence. However, many social sciences also saw religion as a core element of their areas of study and 
eventually tried to expand into the subject as well. The result is a current state of multidisciplinarity, which mirrors the broader structure of social science. Demography, on the other hand, began as a problem-based knowledge project with a relatively unbounded structure and engagement by numerous disciplines and perspectives. As resources began to accumulate and questions narrow, demography - as a field- has grown into a nascent, yet powerful discipline with somewhat porous boundaries.

Focusing on a different dimension of knowledge production, our illustrative examples drawn from HIV/AIDS research describe how boundary work progresses within a field at the problem level. The question of mother-to-child-transmission of HIV was a core concern that initially engaged a flurry of transdisciplinary research. The discovery of nevirapine effectively "solved" this problem and remaining ancillary questions are solved mostly within disciplines. On the other hand, drug resistance remains an open or unresolved question. Research on this question begins in a disciplinary fashion and moves to a multidisciplinary one, perhaps, as a result of the difficulties in advancing knowledge on resistance. Specialization with highly complex biomedical problems may encourage multidisciplinary, but hinder interdisciplinary research. These trajectories serve as several possibilities for the paths that knowledge work can assume in the model that we outline, yet this is not an exhaustive list. Evaluations of knowledge projects should continue to explore the trajectories of successful and failed research programs.

A key tension within prior work on interdisciplinarity examines whether evaluations of interdisciplinarity should focus on ideas-based or organizational concerns. Our multidimensional focus recognizes that these are not mutually exclusive positions. Rather, epistemological and organizational concerns are dimensions of a complex system of boundary work where actors attempt to both solve problems and secure resources. In other words, an institutional approach to 
understanding is not inherently incompatible with an idea-based approach. By seeing these as dimensions of a dynamic system, our theoretical model becomes one basis for evaluating these competing claims. Yet, our current examples draw from the field at the level of problems, at the expense of parallel analyses at the organizational level. A more complete understanding requires the pairing of organizational - and specifically resource-based - data with problem- and fieldlevel data.

Fortunately, methodological tools increasingly parallel the multidimensional and dynamic theory that we propose. Advanced techniques in computer and information science and methods for the dynamic analysis of social networks promise to change the landscape of how we empirically model the organization of knowledge. Those interested in building critical tools for evaluating when interdisciplinarity works and when it fails have a robust theoretical framework and the appropriate methodological tools to work with. Given the entrenched and powerful interests involved, this combination must be leveraged to generate a successful empirical approach to understanding interdisciplinarity. 


\section{References}

Abbott, Andrew. 2001. Chaos of Disciplines. University of Chicago Press.

adams, jimi and Ryan Light. 2014. "Mapping Interdisciplinary Fields: Efficiencies, Gaps \& Redundancies in Hiv/Aids Research." PLoS One forthcoming. doi:

10.1371/journal.pone.0115092.

Bettencourt, Luis M. A., David L. Kaiser, Jasleen Kaur, Carlos Castillo-Chavez and David E. Wojick. 2008. "Population Modeling of the Emergence and Development of Scientific Fields." Scientometrics 75(3):495-518.

Blei, David M., Ng, Andrew Y., and Michael I. Jordan. "Latent Dirichlet Allocation." Journal of Machine Learning 3:993-1022.

Boyack, Kevin W., Richard Klavans and Katy Börner. 2005. "Mapping the Backbone of Science." Scientometrics 64(3):351-74.

Campbell, Donald T. 1969. "Ethnocentrism of Disciplines and the Fish-Scale Model of Omniscience." in Interdisciplinary Relationships in the Social Sciences, edited by M. Sherif and C. W. Sherif. Chicago: Aldine Press.

Delaney, Kevin P., Bernard M. Branson, Apurva Uniyal, Peter R. Kerndt, Patrick A. Keenan, Krishna Jafa, Ann D. Gardner, Denise J. Jamieson and Marc Bulterys. 2006.

"Performance of an Oral Fluid Rapid Hiv-1/2 Test: Experience from Four Cdc Studies." AIDS 20(12):1655-60 10.097/01.aids.0000238412.75324.82.

Dubrow, Joshua Kjerulf. 2011. "Sociology and American Studies: A Case Study in the Limits of Interdiscplinarity." The American Sociologist 42:303-315.

Frodeman, Robert. 2014. Sustainable Knowledge: A Theory of Interdisciplinarity. New York: Palgrave McMillan.

Gieryn, Thomas F. 1999. Cultural Boundaries of Science: Credibility on the Line. Chicago: Chicago University Press.

Griffiths, Thomas L. and Mark Steyvers. 2004. "Finding Scientific Topics." Proceedings of the National Academy of Science 101(S1):5228-35.

Gmur, Markus. 2003. "Co-Citation and the Search for Invisible Colleges: A Methodological Evaluation." Scientometrics 57(1):27-57.

Guay, Laura A., Philippa Musoke, Thomas Fleming, Danstan Bagenda, Melissa Allen, Clemensia Nakabiito, Joseph Sherman, Paul Bakaki, Constance Ducar, Martina Deseyve, Lynda Emel, Mark Mirochnick, Mary Glenn Fowler, Lynne Mofenson, Paolo Miotti, Kevin Dransfield, Dorothy Bray, Francis Mmiro and J. Brooks Jackson. 1999. "Intrapartum and Neonatal Single-Dose Nevirapine Compared with Zidovudine for Prevention of Mother-to-Child Transmission of Hiv-1 in Kampala, Uganda: Hivnet 012 Randomised Trial." The Lancet 354(9181):795-802.

Hadorn, G. Hirsch, Christian Pohl and Gabriele Bammer. 2010. "Solving Problems through Transdisciplinary Research." Pp. 431--52 in The Oxford Handbook of Interdisciplinarity, edited by J. T. Klein and C. Mitcham: Oup Oxford.

Hodgson, Dennis. 1991. "The Ideological Origins of the Population Association of America." Population and Development Review 17(1):1-34. doi: 10.2307/1972350.

Jacobs, Jerry and Scott Frickel. 2009. "Interdisciplinarity: A Critical Assessment." Annual Review of Sociology 35:43-65.

Jacobs, Jerry A. 2013. In Defense of Disciplines: Interdisciplinarity and Specialization in the Research University. Chicago: University of Chicago Press. 
Klein, Julie Thompson. 1990. Interdisciplinarity: History, Theory, and Practice. Detroit, MI: Wayne State University Press.

Kohler, Robert E. 1982. From medical chemistry to biochemistry: The making of a biomedical discipline. Cambridge UP.

Kreager, Philip. 1993. "Histories of Demography: A Review Article." Population Studies 47(3):519-39. doi: 10.1080/0032472031000147286.

Lamont, Michele and Virag Molnar. 2002. "The Study of Boundaries in the Social Sciences." Annual Review of Sociology 28:167-95.

Leydesdorff, Loet and Thomas Schank. 2008. "Dynamic Animations of Journal Maps: Indicators of Structural Changes and Interdisciplinary Developments." Journal of the American Society for Information Science and Technology 59(11):1810-18.

Light, Ryan and jimi adams. 2010. "The Spread of Hiv/Aids Research: Topic Structures in Aids and Jaids, 1988-2008." Paper presented at the Sunbelt XXX - Annual Meetings of the International Network for Social Network Analysis, Riva del Garda, Italy.

Liu, Zao and Chengzhi Wang. 2005. "Mapping Interdisciplinarity in Demography: A Journal Network Analysis." Journal of Information Science 31(4):308-16.

Mayer, Karl Ulrich. 2009. "New Directions in Life Course Research ". Annual Review of Sociology 35:413-33.

Mohr, John W. and Petko Bogdanov. 2013. "Introduction: Topic Models: What they are and why they matter." Poetics 41:545-69.

Moody, James and Ryan Light. 2006. "A View from Above: The Evolving Sociological Landscape." The American Sociologist 37(2):67-86.

Morgan, S. Philip and Scott M. Lynch. 2001. "Success and Future of Demography: The Role of Data and Methods." Annals of the NY Academy of Sciences 954:35-51.

NAS, National Academy of Sciences, National Academy of Engineering, Institute of Medicine and Committee on Facilitating Interdisciplinary Research. 2005. Factilitating Interdisciplinary Research. Washington, DC: National Academies Press.

Newman, Mark. 2008. "The Physics of Networks." Physics Today 61:33-8,

Newman, William M. 1974. "The Society for the Scientific Study of Religion: The Development of an Academic Society." Review of Religious Research 15(3):137-51.

Rojas, Fabio. 2010. From Black Power to Black Studies: How a Radical Social Movement Became and Academic Discipline. Baltimore, MD: Johns Hopkins University Press.

Smilde, David and Matthew May. 2010. "The Emerging Strong Program in the Sociology of Religion." SSRC Working Papers. New York, NY.

Steyvers, M. and T. Griffiths. 2007. "Probabilistic Topic Models." Handbook of Latent Semantic Analysis 427(7):424-40.

Taylor, Mark. 2010. Crisis on Campus: A Bold Plan for Reforming Our Colleges and Universities. New York: Knopf.

Yan, Erija and Ying Ding. 2012. "Sholarly Network Similarities: How Bibliographic Coupling Networks, Citation Networks, Cocitation Networks, Topical Networks, Coauthorship Networks and Coword Networks Relate to Each Other." Journal of the American Society for Information Science and Technology 63(7):1313-26. 
6. The Visibility of Research Dynamics

1. Following Jacobs and Frickel (2009:45), we define multidisciplinarity as the "contributions of two or more fields to a research problem," interdisciplinarity as the "integration of knowledge originating in two or more fields," and transdisciplinarity as "knowledge produced jointly by disciplinary experts and social practitioners." Disciplinarity, on the other hand, is the institutionally bounded, modal form of research production on academic campuses.

2. Note that Freeman (2004) and others try to guide the reception of physics within the networks community and not eliminate cross-pollination. In fact, Freeman encourages bridge-building between these communities. Nonetheless, as an act of turf defense, the resistance of cooptation captures a more modest form of expulsion.

3. The corpus included all articles published from 1960-2009 that are included in the Social Sciences Citation Index that included any of the following as a keyword $(*$ denotes a wildcard search term, such that church* includes church, churches, etc.): church*, relig*, secular*, god*, spiritual $^{*}$, denomination*, congregat*, catholic*, protestant*, faith*, fundamentalis $*$, clerg*. This list was generated from identifying the top 100 keywords used in 2 social science journals that focus on religion: Journal for the Scientific Study of Religion and Review of Religious Research.

4. Those journals represent a wide-range of general research on demography (2012 Impact Factor in Parentheses): Demography (2.305), Population Research and Policy Review (.610), Population Studies (1.147), Population and Development Review (1.998), and Demographic Research (1.047). Due to this sampling, the period examined covers 1956-2012.

5. For an overview of the use of topic models in sociology see Mohr and Bogdanov (2013). 
6. All visualizations require some visual reduction. Here, we included only edges in the network that are especially strong (mean plus two standard deviations). The increasing dominance of the social sciences, as seen in the central position of the yellow nodes in 2008 , is even clearer in the full analyses from which these visualizations are extracted; see Appendices S3 and S4 in adams and Light (2014) for the full details. 
Figure 6.1 A Conceptual Model of Disciplinary Academic Boundary-Work

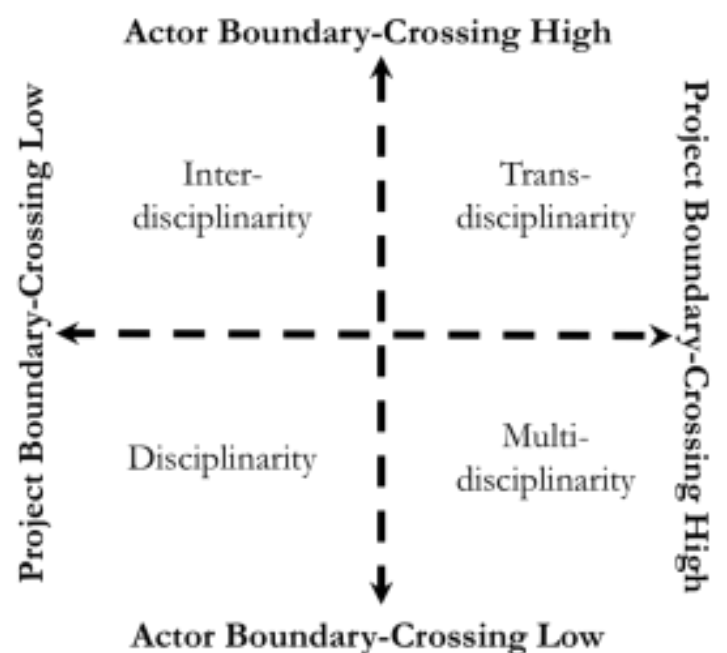


Figure 6.2 Structural Comparison of the Social Sciences of Religion (A) to All Social Sciences (B)

A

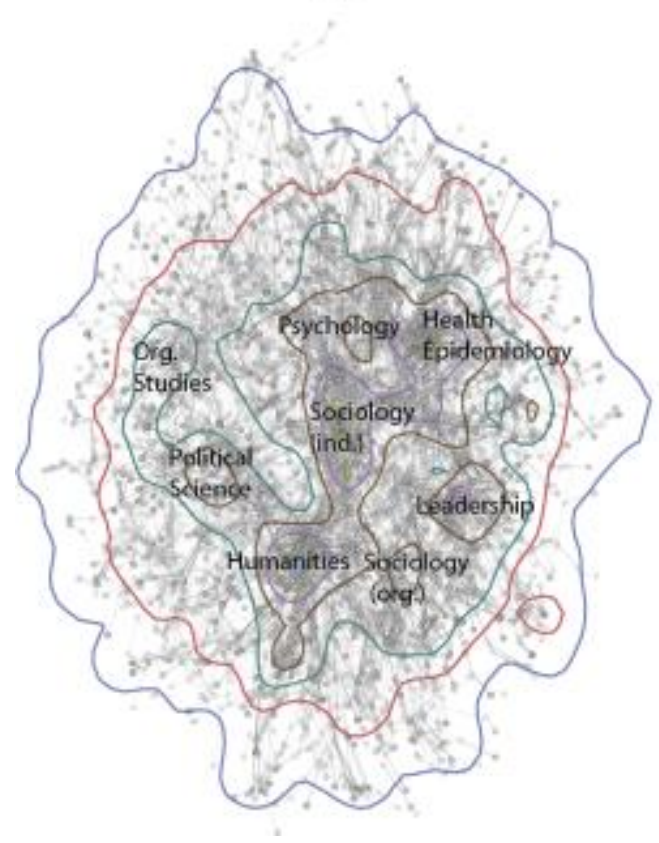

B

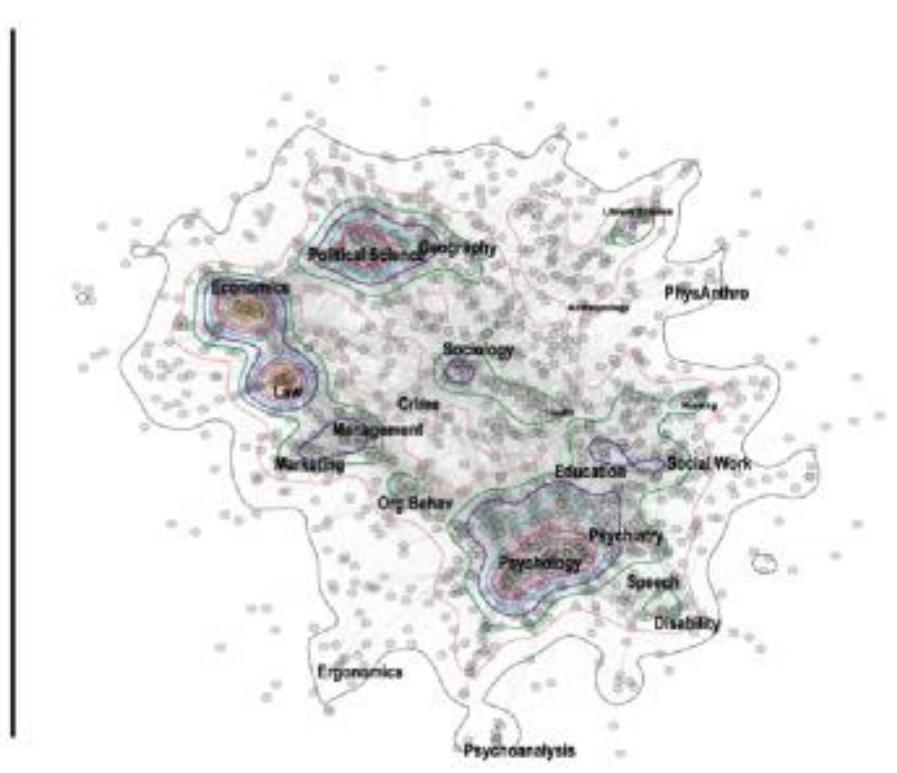


Figure 6.3 Consolidation in PMTCT Research

1997

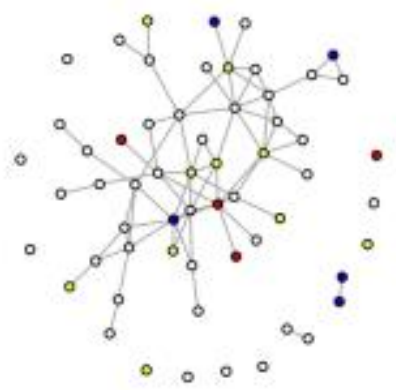

2005

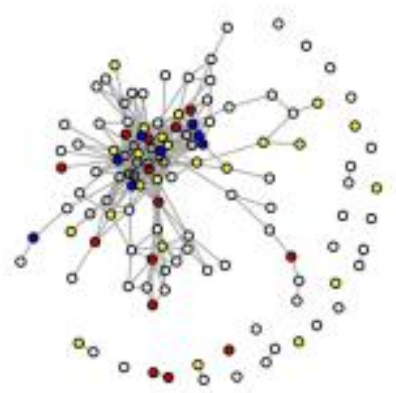

2001

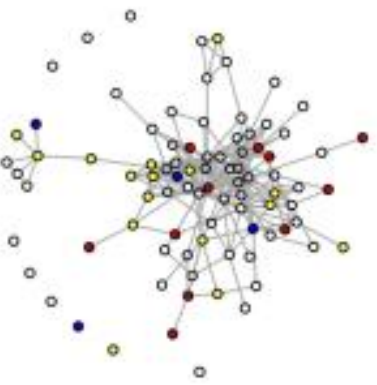

2008

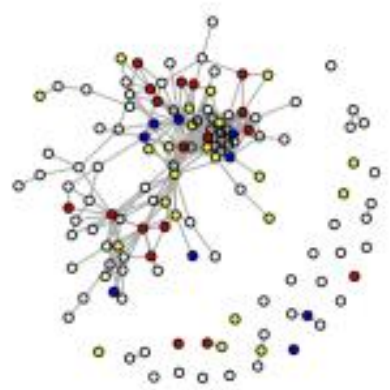


Figure 6.4 Segmented Communities in Drug Resistance HIV Research, 2003

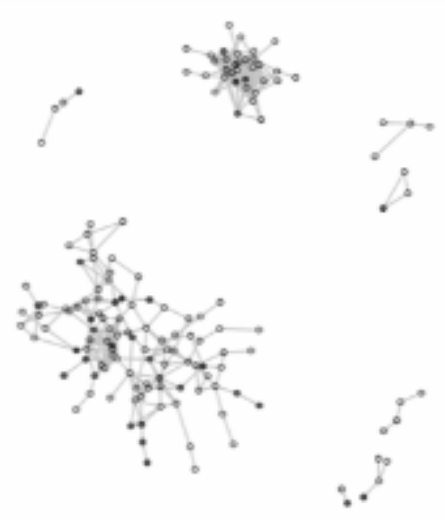

2003 